\title{
Creep, Hysteresis, and Vibration Effects Attenuation in an AFM PTS
}

\author{
M. S. Rana, Student Member, IEEE, H. R. Pota, Member, IEEE, and I. R. Petersen, Fellow, IEEE
}

\begin{abstract}
There is a need, in the wide ranging scientific community, to perform fast scans using atomic force microscope (AFM) with nanoscale accuracy. The performance of AFM at high scanning speeds is limited due to some serious limitations of its scanning unit; i.e., the piezoelectric tube scanner (PTS). In order to increase the imaging speed of an AFM, a multi-input multi-output (MIMO) model predictive control (MPC) scheme is applied to the axes of the PTS to reduce its creep, hysteresis, and vibration effects. The design of this controller is based on an identified MIMO model of the AFM PTS. Also, a vibration compensator is designed and included in the feedback loop with the plant to suppress the vibration of the PTS at the resonant frequency. To evaluate the performance improvement achieved using the proposed control scheme, experimental comparisons of the scanned images obtained using open-loop and the in-built proportional-integral (PI) controller is made. The comparison demonstrates the usefulness of the proposed controller.
\end{abstract}

\section{INTRODUCTION}

Precise positioning on a nanometric scale, often referred to as nanopositioning, is a key enabling technology for nanoscale science and engineering. One of the major techniques in nanotechnology that relies heavily on nanopositioning is the atomic force microscope (AFM). It enables precise control, manipulation, and interrogation of matter at the nanoscale [1]. It was invented by Binnig et al. [2], has the ability to generate three-dimensional images of material surfaces at an extremely high resolution down to the atomic level $\left(10^{-10} \mathrm{~m}\right)$. It is extensively used in various areas such as nanolithiography, DNA nanotechnology, optics, microelectronics, material science, and nanofabrication [3], [4].

In an AFM arrangement, to control the tip-sample distance and move the tip over the sample surface with high accuracy (at a level of Angstrom fractions), a special type of transducer or scanning element called a piezoelectric tube scanner (PTS) is used. The main features of the PTS are that it can generate a large force in a range of temperatures, repeats subnanometer motion without backlash, is generally free of wear and tear, has a fast response time, requires little maintenance, and is normally not affected by magnetic fields. In spite of many useful properties, there are some challenges associated with the use of the PTS for precision positioning. The most prominent limitations of the PTS are the low mechanical resonance frequency, non-linear behavior in the form of hysteresis and creep effects, and cross coupling between the axes.

M. S. Rana, H. R. Pota, and I. R. Petersen are with the School of Engineering and Information Technology, The University of New South Wales, Canberra, ACT 2600, Australia (e-mail: m.rana@student.unsw.edu.au; h.pota@adfa.edu.au; i.petersen@adfa.edu.au).

This work was supported by the Australian Research Council (ARC).

978-3-9524269-1-3 @ EUCA

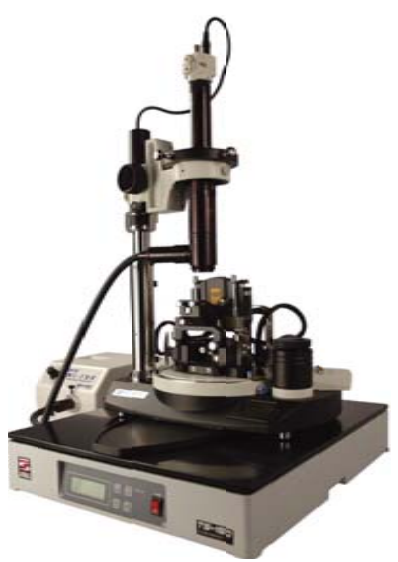

Fig. 1. NT-MDT Ntegra scanning probe microscope arranged to operate as an AFM.

During the operation of the PTS, positioning defects arise due to creep (or drift), when the PTS is actuated over extended periods of time (e.g., during slow speed scanning operations). Hysteresis, a nonlinear behavior of PTS which become noticeable when it is actuated over a long range of operation. The most serious problem with the PTS is induced vibration, when it is actuated at a high frequency. Feedback control techniques to improve the accuracy and speed of the AFM have been reported in the literature [5], [6]. Creep, hysteresis, and vibration effects are minimized by implementing a proportional plus derivative high-gain feedback controller and feed-forward controller in [5]. In [7] creep compensator of Preisach type is used and its performance has been evaluated in real time application which achieved a significant compensation of creep effect.

In [8] the design of an ILC algorithm is presented to achieve high precision of an AFM system which is based on the Preisach hysteresis model. A frequency based hysteresis compensation for PTS using ANNs is presented in [9] which achieved better compensation of hysteresis effect, but at a small value of frequency of $1 \mathrm{~Hz}$. This is in order to avoid vibration problem at high frequencies due to excitation of the tube resonant. In [10] a novel hysteresis operator and the development of a rate-independent (RI) hysteresis model and a rate-dependent (RD) hysteresis model for a piezoelectric actuator (PEA) one-sided hysteresis is presented, which achieved a significant compensation of hysteresis effect.

The lightly damped resonant modes in the PTS create significant distortions in the AFM's scanned images [6], [11]. The fast axis, i.e., the $X$-axis of the PTS is driven by a triangular signal and the slow axis, i.e., the $Y$-axis is driven by a slowly increasing staircase signal or the ramp signal [12]. This 


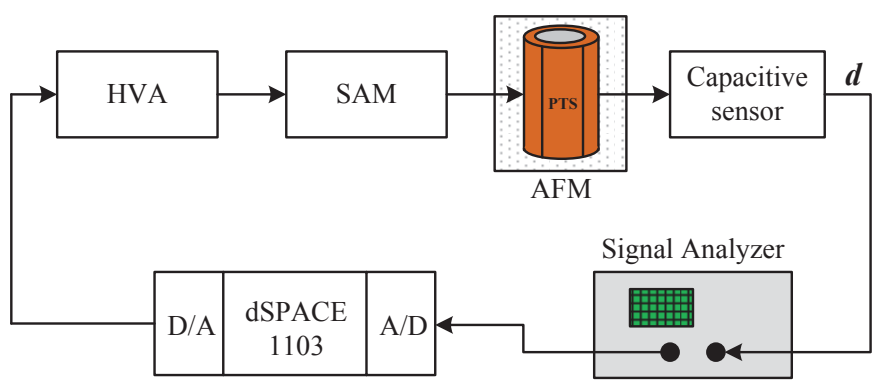

Fig. 2. Schematic diagram of the experimental setup.

triangular signal contains odd harmonics of the fundamental frequency which cause a fast triangular signal to excite the resonance of the PTS for which the resulting generated images at high scanning rates are distorted. To suppress the resonant mode of the AFM PTS, a positive velocity and position feedback (PVPF) controller is proposed by Bhikkaji et al. in [13].

A better tracking of the reference signal ensures compensation of the creep and hysteresis effects in the PTS. An MPC control scheme is commonly used when tracking a reference trajectory is the primary goal. It has been proven to be a successful control technique in a diversity of devices and procedures, ranging from robot manipulators [14] to clinical anaesthesia [15], where such tracking is a key requirement. This capability of MPC controllers has motivated our research to compensate the creep, hysteresis, and vibration effects of the PTS to improve the imaging performance of AFMs. To achieve this a multi-input multi-output (MIMO) MPC scheme is designed to counteract creep and hysteresis effects, and using a vibration compensator to improve the damping of the resonant mode without modeling the system's complicated nonlinear behavior.

The paper is organized as follows: Section II presents the descriptions of the AFM and the experimental setup used in this work. Section III provides the modeling and identification of the system transfer functions. In Section IV, the control scheme for the AFM scanner is presented. Section V reflects the experimental results which illustrates the effectiveness of the proposed control scheme. Finally, Section VI discusses the conclusions.

\section{EXPERIMENTAL SETUP}

The experimental setup at the University of New South Wales (UNSW), Canberra, Australia, consists of an NT-MDT Ntegra scanning probe microscope (SPM), configured to operate as an AFM (see Fig. 1). It contains a signal access module (SAM), control electronics, vibration isolator, computer for operating the AFM NOVA software, and other accessories, that is, a dynamic signal analyzer (DSA), a DSP dSPACE RT1103 , and a high voltage amplifier (HVA) with a constant gain of 15 for supplying power to the $X, Y$, and $Z$-piezos using the SAM as an intermediate device. In this work, a Sm8133cl type scanner is used which is a "scan by head" type of scanner. A block diagram of the experimental setup is shown in Fig. 2.

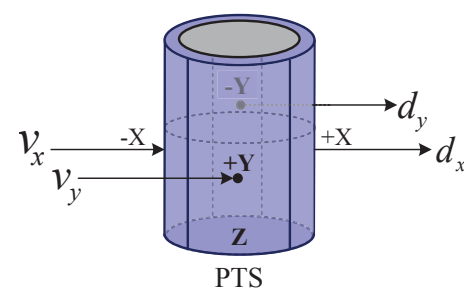

Fig. 3. I/O diagram of the AFM PTS.
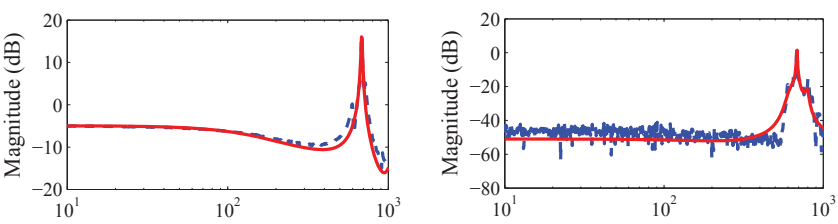

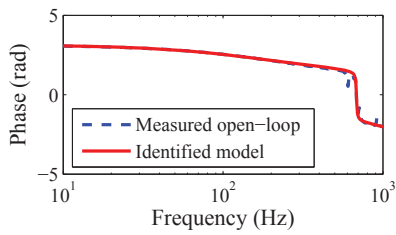

(a) $G_{x x}$
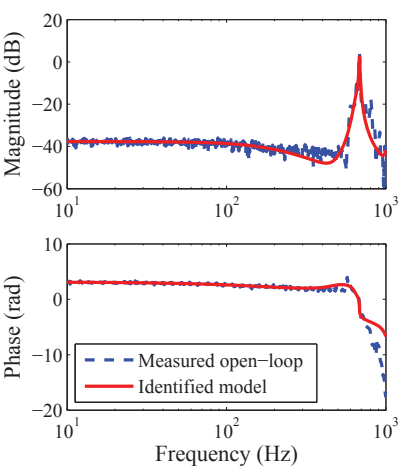

(c) $G_{y x}$

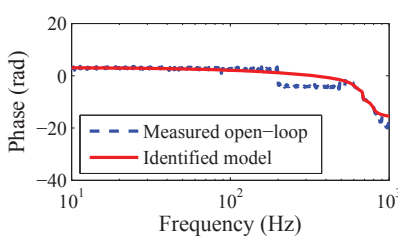

(b) $G_{x y}$
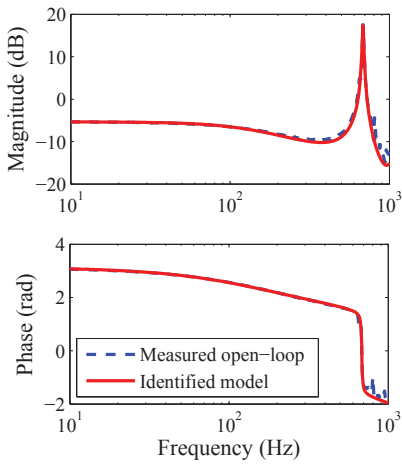

(d) $G_{y y}$
Fig. 4. Frequency responses of the measured and identified systems for (a) input to the $X$ piezo and output from the $X$ position sensor, (b) input to the $Y$ piezo and output from the $X$ position sensor, (c) input to the $X$ piezo and output from the $Y$ position sensor, and (d) input to the $Y$ piezo and output from the $Y$ position sensor.

\section{IDENTIFICATION OF THE PTS DYNAMICS}

In this work the PTS is modeled as a two-input two-output (TITO) system (see Fig. 3) and the plant is identified using a bandlimited random noise signal within the frequency range from $10 \mathrm{~Hz}$ to $1.0 \mathrm{kHz}$, using a dual channel HP35665A DSA. This signal is supplied to the HVA as an input and the corresponding amplified voltage is supplied to the SAM of the AFM from which there is a direct connection to the PTS. The output displacement of the PTS is taken from the capacitive position sensor. The sensor output is fed back to the DSA to obtain frequency response functions (FRFs). The FRFs generated in the DSA are processed in MATLAB and using prediction error method (PEM), a system model is obtained. The best fit model frequency responses for the $X$ and $Y$-piezos are shown in Fig. 4.

The two inputs are the voltages applied to the $x$ and $y$ - 


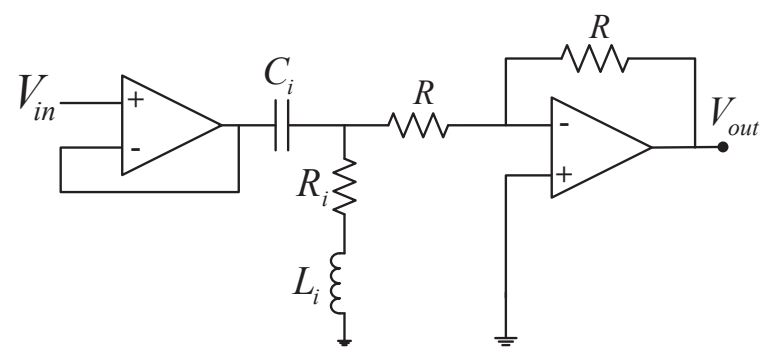

Fig. 5. Structure of the vibration compensator.

axes amplifiers $\left[v_{x}, v_{y}\right]^{T}$ while the corresponding output from the capacitive sensors $\left[d_{x}, d_{y}\right]^{T}$. The FRFs of the AFM lateral positioning system can be described by the following equation

$$
G_{d v}(s)=\left[\begin{array}{ll}
\frac{d_{x}(s)}{v_{x}(s)} & \frac{d_{x}(s)}{v_{y}(s)} \\
\frac{d_{y}(s)}{v_{x}(s)} & \frac{d_{y}(s)}{v_{y}(s)}
\end{array}\right]
$$

where

$\frac{d_{x}(s)}{v_{x}(s)}=\frac{-1.197 \times 10^{4} s^{3}-2.289 \times 10^{6} s^{2}+1.205 \times 10^{9} s-1.599 \times 10^{13}}{s^{4}+3.859 \times 10^{5} s^{3}+6.626 \times 10^{7} s^{2}+1.806 \times 10^{11} s+2.849 \times 10^{13}}(2)$

$\frac{d_{x}(s)}{v_{y}(s)}=\frac{4.242 s^{3}-2460 s^{2}+2.682 \times 10^{6} s-8.622 \times 10^{8}}{s^{4}+95.6 s^{3}+1.016 \times 10^{6} s^{2}+4.498 \times 10^{7} s+2.56 \times 10^{11}}$

$\frac{d_{y}(s)}{v_{x}(s)}=\frac{0.9309 s^{3}-5498 s^{2}+2.551 \times 10^{6} s-2.692 \times 10^{9}}{s^{4}+379.8 s^{3}+9.67 \times 10^{5} s^{2}+1.772 \times 10^{8} s+2.327 \times 10^{11}}$

and

$\frac{d_{y}(s)}{v_{y}(s)}=\frac{-40.86 s^{3}+2.703 \times 10^{4} s^{2}-1.363 \times 10^{7} s-6.895 \times 10^{10}}{s^{4}+1.763 \times 10^{3} s^{3}+7.812 \times 10^{5} s^{2}+8.214 \times 10^{8} s+1.316 \times 10^{11}}$

\section{Controller Design}

\section{A. Design of Vibration Compensator}

This section presents the design of a vibration compensator, the basic structure of which is shown in Fig. 5. Although the MPC controller has itself some damping capacity, a vibration compensator is introduced to achieve better damping of the resonant mode. From the experimental frequency responses of the PTS, we know that the first resonance occurs at approximately $681.50 \mathrm{~Hz}$ for both the $X$ and $Y$-piezos at loaded condition. The vibration compensator is designed to damp the first resonant mode with a bandwidth close to the PTS's first resonant frequency. The general form of the vibration compensator is [16]:

$$
A_{i}=\sum_{i=1}^{N}-k_{i} \frac{C_{i} s\left(R_{i}+L_{i} s\right)}{L_{i} C_{i} s^{2}+R_{i} C_{i} s+1}
$$

where $i=1,2, \cdots, N, k_{i}$ is the compensator gain of the corresponding mode. By selecting the proper value of $L_{i}, R_{i}$, and $C_{i}$, we are able to improve damping of the resonant mode of the PTS. Since $\omega_{i}=\frac{1}{\sqrt{L_{i} C_{i}}}$, the value of $L_{i}$ and $C_{i}$ are chosen such that $\omega_{i}$ is equal to or almost equal to the resonant frequency of the system.

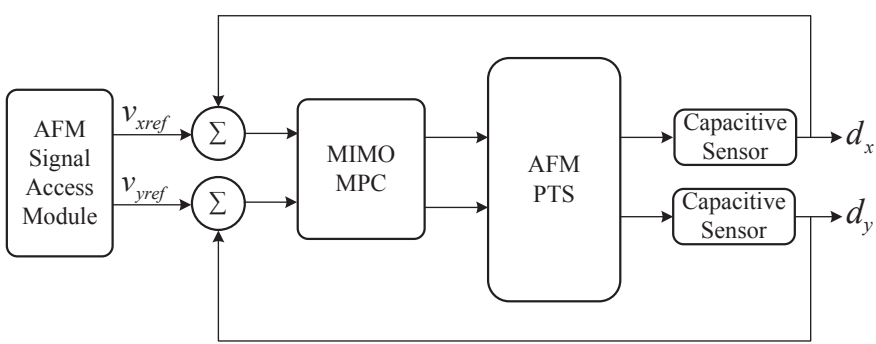

Fig. 6. Block diagram of the closed-loop system. $v_{x r e f}$ and $v_{y r e f}$ are the scanning reference waveforms provided by the AFM signal access module. The outputs $d_{x}$ and $d_{y}$ are the displacements of the tube scanner.

\section{B. Design of MIMO MPC}

The purpose of this section to present the design of an MIMO MPC controller for minimizing different nonlinearity effects in the AFM PTS. The construction of this closed-loop system is shown in Fig. 6.

Now-a-days, MPC is considered to be one of the most widely used multivariable control algorithms in a variety of devices. The MPC scheme is employed to design a tracking controller, the output from which tracks a reference input. It consists of a reference block, a predictor block, and an optimizer block [17]. The reference block provides the future reference trajectory which is used to compute the cost function using the predictions from the predictor block. This cost function is then optimized in the optimizer block. The plant is described by the following state-space model:

$$
\begin{aligned}
x_{m}(k+1) & =A_{m} x_{m}(k)+B_{m} u(k) \\
y(k) & =C_{m} x_{m}(k)
\end{aligned}
$$

(5) where $A_{m}, B_{m}$, and $C_{m}$ define the discrete state-space plant model, derived from the identified plant model, as stated in Eqs. (2)-(5) and Eq. (6) at a sampling time $T_{s}, u=\left[v_{x}, v_{y}\right]^{T}$ is the manipulated variable or input variable, $y=\left[d_{x}, d_{y}\right]^{T}$ is the measured output, and $x_{m}$ is the state variable vector with a dimension of $n$. In order to incorporate integral action for disturbance rejection and tracking a reference signal in the MPC algorithm, the plant can be augmented in the following way [18], [19]:

$$
\begin{gathered}
\overbrace{\left[\begin{array}{c}
\Delta x_{m}(k+1) \\
y(k+1)
\end{array}\right]}^{x(k+1)}=\overbrace{\left[\begin{array}{cc}
A_{m} & 0 \\
C_{m} A_{m} & I
\end{array}\right]}^{A} \overbrace{\left[\begin{array}{c}
\Delta x_{m}(k) \\
y(k)
\end{array}\right]}^{x(k)} \\
+\overbrace{\left[\begin{array}{c}
B_{m} \\
C_{m} B_{m}
\end{array}\right]}^{B} \Delta u(k) \\
y(k)=\overbrace{\left[\begin{array}{ll}
0 & I
\end{array}\right]}^{C} \overbrace{\left[\begin{array}{c}
\Delta x_{m}(k) \\
y(k)
\end{array}\right]}^{x(k)}
\end{gathered}
$$

where $A, B$, and $C$ are the augmented system matrices.

The output sequence for $N_{p}$, prediction horizon can be written as:

$$
Y=F x(k)+\Phi \Delta U
$$


in which

$$
Y=\left[\begin{array}{c}
y(k+1 \mid k) \\
y(k+2 \mid k) \\
\vdots \\
y\left(k+N_{p} \mid k\right)
\end{array}\right] ; \Delta U=\left[\begin{array}{c}
\Delta u(k) \\
\Delta u(k+1) \\
\vdots \\
\Delta u\left(k+N_{c}-1\right)
\end{array}\right] .
$$

where $N_{c}$ is the control horizon, and the $F$ matrix with dimensions of $\left(2 N_{p}, n\right)$ and the $\Phi$ matrix with dimensions of $\left(2 N_{p}, 2 N_{c}\right)$ are:

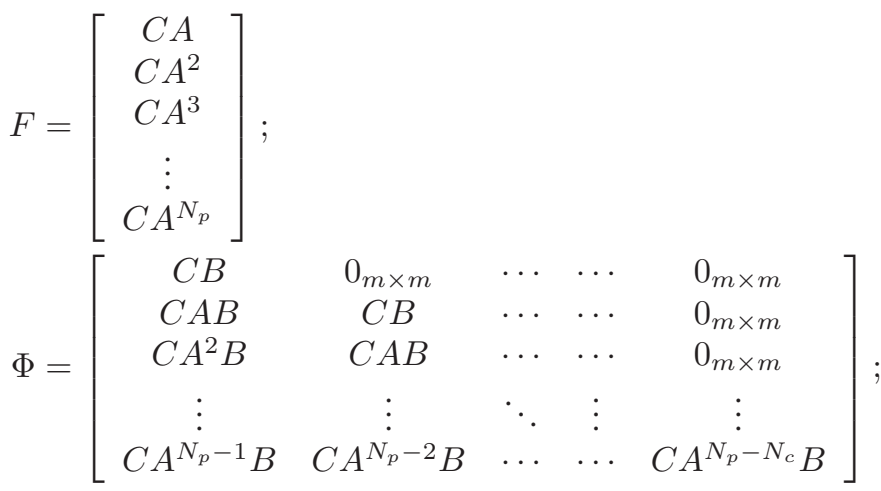

where in $0_{m \times m}, \mathrm{~m}$ is the number of outputs.

The control law is derived based on the minimization of the cost function defined as:

$$
\begin{aligned}
J= & \sum_{m=1}^{N_{p}} Q\left(y(k+m \mid k)-R_{s}(k+m)\right)^{2} \\
& +\sum_{m=1}^{N_{c}} R(\Delta u(k+m-1))^{2}
\end{aligned}
$$

subject to the linear inequality constraints on the system inputs, i.e:

$$
\begin{aligned}
u_{\min } \leq u(k+i-1) \leq u_{\max }, & i=1, \ldots, N_{c} \\
\Delta u_{\min } \leq \Delta u(k+i-1) \leq \Delta u_{\max }, & i=1, \ldots, N_{c}
\end{aligned}
$$

where $Q$ is the state weighting matrix, $R$ is the control weighting, $R_{s}$ is the reference signal, $u_{\min }$ and $u_{\max }$ are the low and high levels of the control action, respectively, and $\Delta u_{\min }$ and $\Delta u_{\max }$ are the low and high levels of the control increments, respectively.

By considering the above equations, the constrained MPC problem can be expressed as a quadratic programming (QP) problem:

$$
\begin{gathered}
\min \left(\frac{1}{2} \Delta U^{T} H \Delta U+\Delta U^{T} f\right) \\
\text { s.t. } \quad M \Delta U \leq \gamma ;
\end{gathered}
$$

where

$$
\begin{aligned}
H & =\Phi^{T} Q \Phi+R ; \\
f & =\Phi^{T} Q F x(k+1 \mid k)-\Phi^{T} Q R_{s} ;
\end{aligned}
$$

$M \in \mathbb{R}^{m_{c} \times 2 N_{c}}$ and $\gamma \in \mathbb{R}^{2 N_{c} \times 1}$ are computed using Eq. (13), $m_{c}$ is the number of constraints and $R_{s} \in \mathbb{R}^{2 N_{p} \times 1}$ is the reference signal. In this paper for constraint calculation the Hildreth's QP algorithm has been considered. The constrained minimization over $\Delta U$ is given by [18]

$$
\Delta U=-H^{-1}\left(f+M^{T} \lambda\right)
$$
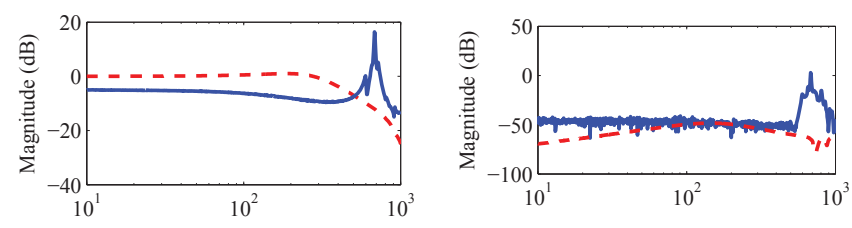

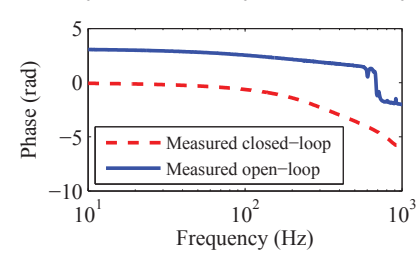

(a)
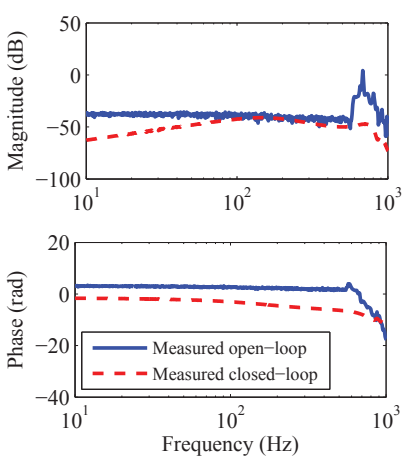

(c)

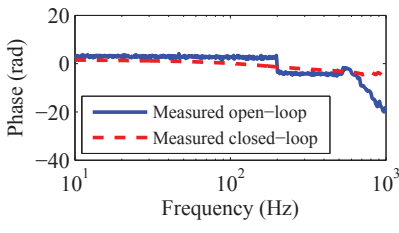

(b)
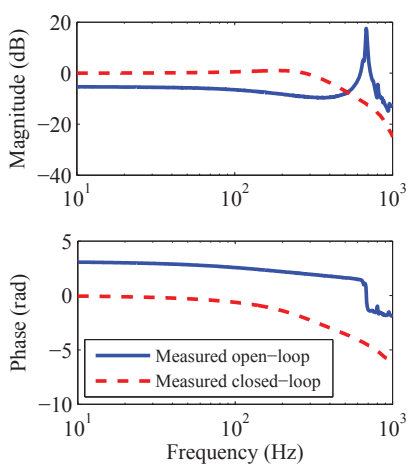

(d)
Fig. 7. Comparison of measured open-loop and closed-loop frequency responses for: (a) input to the $X$-piezo and output from the $X$ position sensor, (b) input to the $Y$-piezo and output from the $X$ position sensor, (c) input to the $X$-piezo and output from the $Y$ position sensor, and (d) input to the $Y$-piezo and output from the $Y$ position sensor.

where $\lambda$ is the Lagrange multiplier, which is calculated by using $M, \gamma, H$, and $f$. The Kalman state observer estimates the states from the measured output and dynamics are:

$$
\begin{aligned}
\hat{x}(k+1) & =(A-L C) \hat{x}(k)+B u(k)+L y(k) \\
\hat{y}(k) & =\hat{C} \hat{x}(k)
\end{aligned}
$$

where $\hat{x}(k)$ is the estimated state, $\hat{y}(k)$ is the estimated output, $\hat{C}$ is the identity matrix of dimension $n \times n$, and $L$ is the observer gain which depends on the Gaussian white noise, process noise covariance, and the measurement noise covariance.

\section{EXPERIMENTAL RESUlTS}

As stated earlier, that the uncontrolled resonant mode is one of the major limiting factors of PTS in its nanopositioning performance. This uncontrolled resonant mode creates a significant vibration in the scanned image and it's severe at higher scanning speeds. To overcome this problem the proposed MIMO MPC controller with a vibration compensator is implemented in the AFM PTS, and a frequency domain analysis is carried out by comparing the measured open-loop and closedloop frequency responses as shown in Fig. 7. Figure 7(a) and (d) show comparisons of the closed-loop frequency plots of the $X$ and $Y$-piezos obtained by implementing the MIMO MPC controller with the vibration compensator, which indicate that it achieves high closed-loop bandwidths of 494 and $503 \mathrm{~Hz}$, 


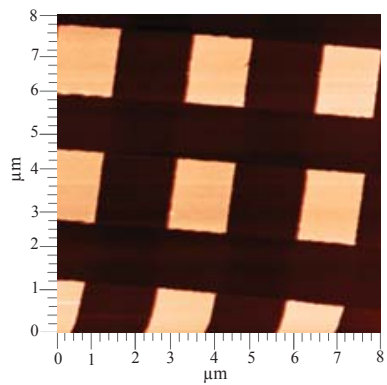

(a)

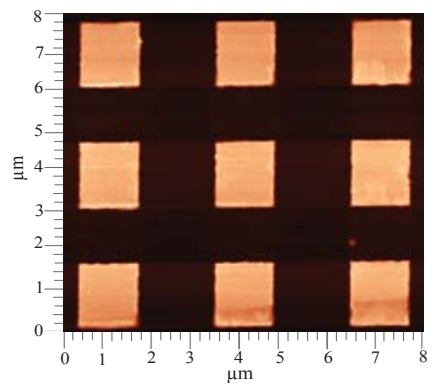

(b)
Fig. 8. Creep effect in the scanned image at the open-loop condition (a) and reduction of creep effect using the proposed MIMO MPC controller (b).

and 23.28 and $24.88 \mathrm{~dB}$ damping of the resonant mode for $X$ and $Y$-piezos, respectively, in turn, significantly reduces vibrations.

The frequency responses for the cross-coupling effects of the AFM PTS are measured and illustrated in Fig. 7(b) and (c). In open-loop, significant cross-coupling effect can be observed between lateral axes of the scanner, and the effect is higher at and close to the resonance frequency of the PTS. In closedloop a substantial reduction in the cross-coupling between the lateral axes of the scanner is achieved and is around $55 \mathrm{~dB}$ for both the axes at closed the resonance frequency. Thus, the controller reduces oscillation and vibration in the system.

During the slow operation of the AFM at open-loop the scanned image has got creep effect as shown in Fig. 8 (a). Due to this effect right side bottom edge of the vertical axis of the scanned image is rolled-off. In Fig. 8(b), the creep effect is reduced significantly using the proposed controller.

The effectiveness of the proposed controller against the hysteresis and vibration effect is depicted in Fig. 9. At openloop condition, because of the uncontrolled tube resonance the scanned images have got vibration and hysteresis effect and the effects are severe at higher scanning speed as shown in Fig. 9(a), (d), (g), \& (j) for scanning speed of 15.62, $31.25,62.50$, and $125 \mathrm{~Hz}$, respectively. Fig. 9 illustrates the results obtained by implementing the proposed controller which shows significantly different images from those taken by the existing AFM PI controller. Their image qualities are nearly the same at $15.62 \mathrm{~Hz}$ although the image from the existing PI controller has a distinct curvature in its horizontal axis because of the hysteresis effect as shown in Fig. 9(b). However, at higher frequencies, such as 31.25, 62.50, and $125 \mathrm{~Hz}$, the scanned images using the existing PI controller have more roll-off error, i.e., hysteresis and vibration while those up to $125 \mathrm{~Hz}$ using the proposed controller retain good quality. Nonetheless, although the scanned image at $125 \mathrm{~Hz}$ from the proposed controller is far better than that from the PI controller, it has some noticeable roll-off error and vibration due to vibration of the cantilever tip.

\section{CONCLUSION}

In this article, results from a study of the high-precision positioning of an AFM PTS using an MIMO MPC controller augmented with a vibration compensator are reported. The closed-loop frequency-domain performance is compared with the open-loop frequency responses and is shown to achieve significant damping of the resonant mode of the PTSs and to reduce creep, hysteresis, and vibration effects. The experimental results show improved scanning performance of the proposed controller at high scanning speed than at the openloop condition and the existing AFM PI controller.

\section{REFERENCES}

[1] B. J. Kenton, A. J. Fleming, and K. K. Leang, "Compact ultra-fast vertical nanopositioner for improving scanning probe microscope scan speed," Review of Scientific Instruments, vol. 82, no. 12, pp. $123703-$ $123703-8$, Dec. 2011.

[2] G. K. Binnig, C. F. Quate, and C. Gerber, "Atomic force microscope (AFM)," Physical Review Letters, vol. 56, no. 9, pp. 930-933, Mar. 1986.

[3] M. S. Rana, H. R. Pota, I. R. Petersen, and Habibullah, "Improvement of the tracking accuracy of an AFM using MPC," in 8th IEEE Conference on Industrial Electronics and Applications (ICIEA), 2013, pp. 16811686.

[4] M. S. Rana, H. R. Pota, and I. R. Petersen, "Performance of sinusoidal scanning with MPC in AFM imaging," IEEE/ASME Transactions on Mechatronics, 2014, [Preprint available online] DOI:10.1109/TMECH.2013.2295112.

[5] K. Leang and S. Devasia, "Feedback-linearized inverse feedforward for creep, hysteresis, and vibration compensation in AFM piezoactuators," IEEE Transactions on Control Systems Technology, vol. 15, no. 5, pp. 927-935, Sep. 2007.

[6] B. Bhikkaji and S. O. R. Moheimani, "Integral resonant control of a piezoelectric tube actuator for fast nanoscale positioning," IEEE/ASME Trans. on Mechatronics, vol. 13, no. 5, pp. 530-537, Oct. 2008.

[7] K. Kuhnen and P. Krejci, "Compensation of complex hysteresis and creep effects in piezoelectrically actuated systems - a new preisach modeling approach," IEEE Transactions on Automatic Control, vol. 54, no. 3, pp. 537-550, 2009

[8] K. K. Leang and S. Devasia, "Design of hysteresis-compensating iterative learning control for piezo-positioners: Application to atomic force microscopes," Mechatronics, vol. 16, pp. 141-158, 2006.

[9] Y. Othman, I. A. Mahmood, A. Aibinu, and N. K. A. M. Rashid, "Frequency based hysteresis compensation for piezoelectric tube scanner using artificial neural networks," Procedia Engineering, vol. 41, pp. 757$763,2012$.

[10] J. Y. Peng and X. B. Chen, "Novel models for one-sided hysteresis of piezoelectric actuators," Mechatronics, vol. 22, pp. 757-765, 2012.

[11] M. S. Rana, H. R. Pota, I. R. Petersen, and Habibullah, "Spiral scanning of atomic force microscope for faster imaging," in IEEE 52nd Annual Conference on Decision and Control (CDC), Dec. 2013, pp. 354-359.

[12] Y. K. Yong, K. Liu, and S. O. R. Moheimani, "Reducing cross-coupling in a compliant XY nanopositioner for fast and accurate raster scanning," IEEE Transactions on Control Systems Technology, vol. 18, no. 5, pp. 1172-1179, Sep. 2010

[13] B. Bhikkaji, M. Ratnam, A. J. Fleming, and S. O. R. Moheimani, "Highperformance control of piezoelectric tube scanners," IEEE Transactions on Control Systems Technology, vol. 15, no. 5, pp. 853-866, Sep. 2007.

[14] D. A. Linkers and M. Mahfonf, Advances in Model-based predictive control, chapter Generalised predictive control in clinical anaesthesia. Oxford University Press, 1994

[15] M. A. Lelic and P. E. Wellstead, "Generalized pole placement self tuning controller. Part 1. basic algorithm." Int. J. Control, vol. 46, no. 2, pp. 547-568, Feb. 1987.

[16] H. R. Pota, S. O. R. Moheimani, and M. Smith, "Resonant controller for smart structures," Smart Materials and Structures, vol. 11, pp. 1-8, Feb. 2002.

[17] M. S. Rana, H. R. Pota, and I. R. Petersen, "High-speed AFM image scanning using observer-based MPC-Notch control," IEEE Transactions on Nanotechnology, vol. 12, no. 2, pp. 246-254, Mar. 2013.

[18] L. Wang, Model Predictive Control System Design and Implementation Using MATLAB. London, UK: Springer, 2009.

[19] M. S. Rana, H. R. Pota, and I. R. Petersen, "Spiral scanning with improved control for faster imaging of AFM," IEEE Transactions on Nanotechnology, 2014, [Preprint available online] DOI:10.1109/TNANO.2014.2309653. 


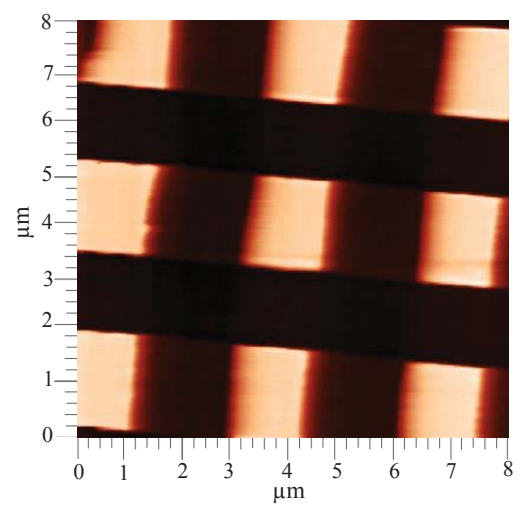

(a)

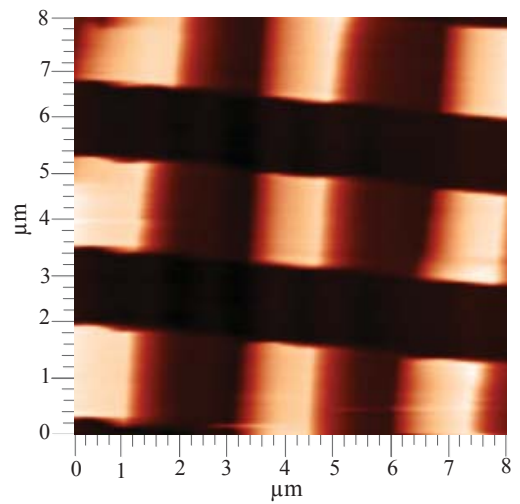

(d)

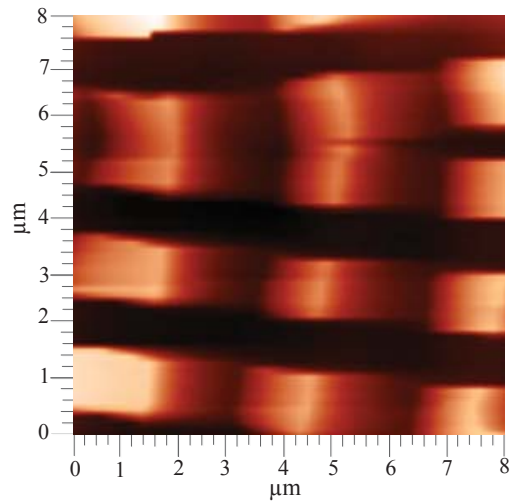

(g)

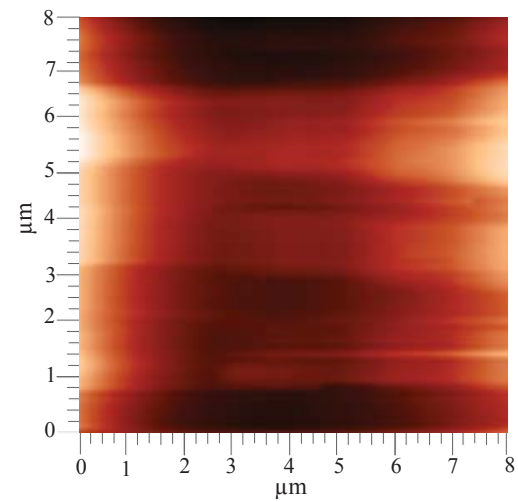

(j)

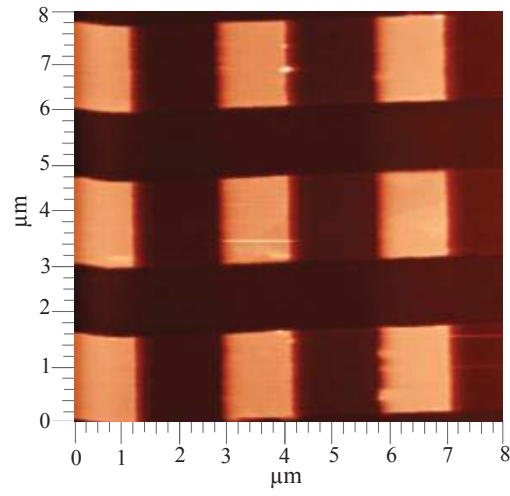

(b)

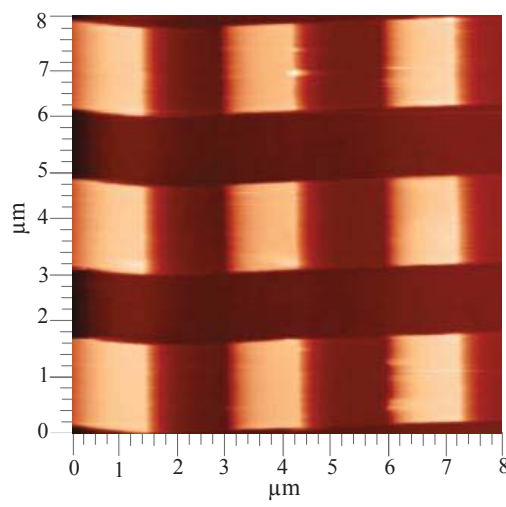

(e)

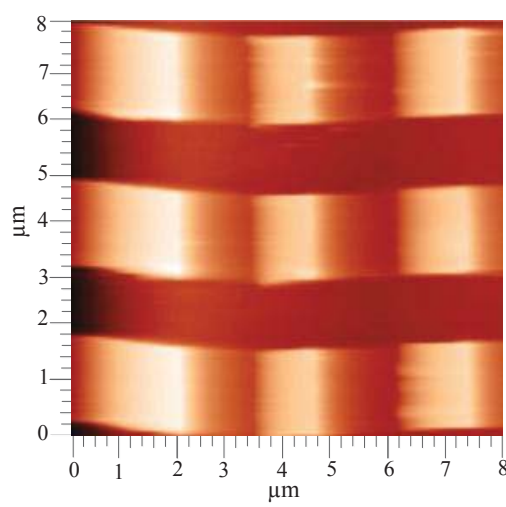

(h)

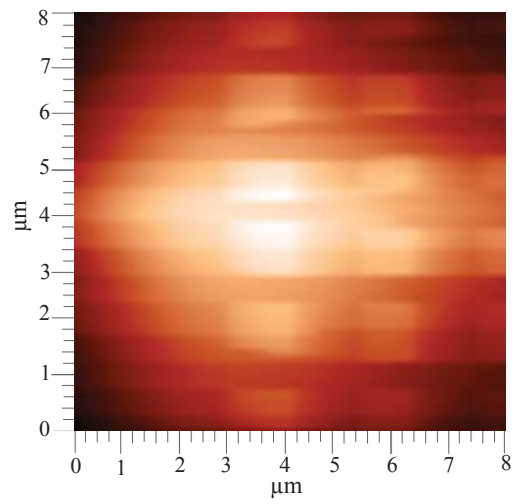

(k)

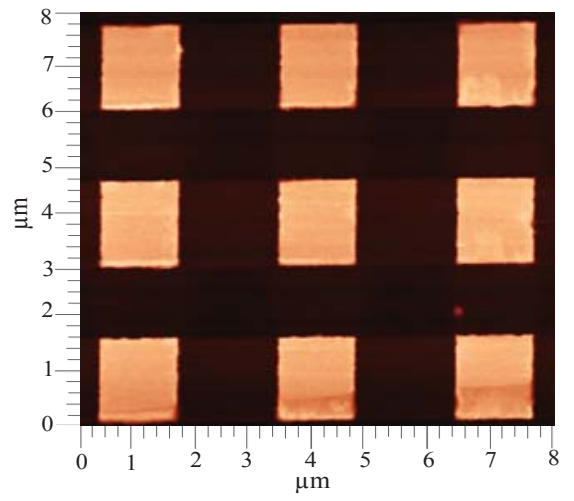

(c)

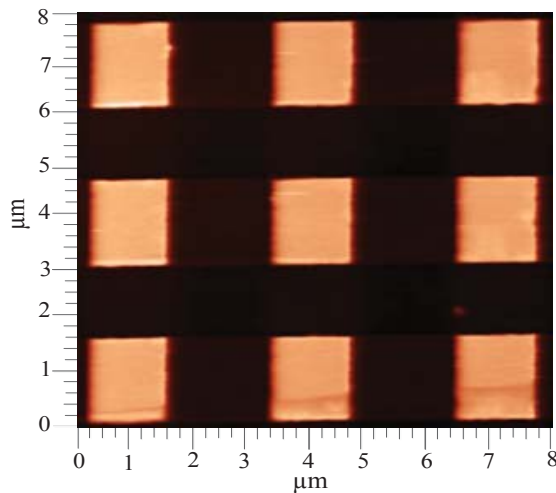

(f)

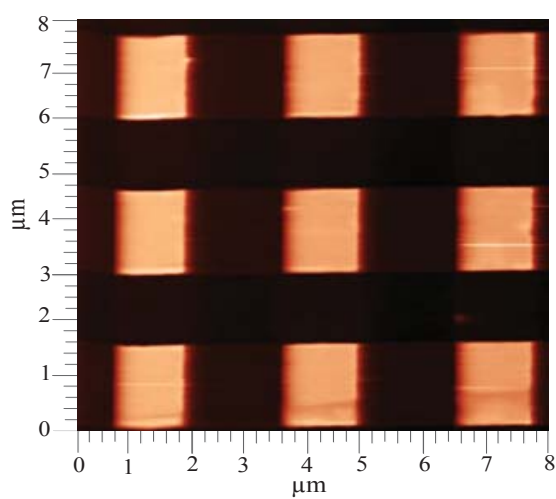

(i)

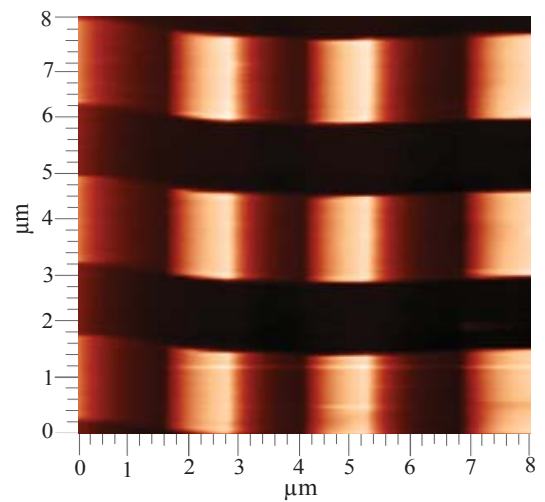

(1)

Fig. 9. Compensation of hysteresis and vibration effects at 15.62, 31.25, 62.50, and $125 \mathrm{~Hz}$ at the open-loop condition ((a), (d), (g), \& (j)); using the AFM PI controller ((b), (e), (h), \& (k)); and using the proposed controller ((c), (f), (i), \& (1)). 